\title{
Design Mechanism: A Design Methodology for the Construction of Urban Reality
}

\author{
Eirini Krasaki \\ National Technical University of Athens, Athens, Greece
}

\begin{abstract}
The proposed design methodology combines data analysis, algorithmic design, linguistics, mathematics physics to analyze methodologically the complex system of the urban fabric. The implementation of this design mechanism uses the urban fabric of Eleusis as a paradigm. The urban body of Eleusis is defined as a palimpsest of descriptions and images, synthesized spatially in multiple layers and is therefore considered to be a suitable sample for the preliminary application of the proposed methodology. Urban reality can be described as a complex system as it consists of both tangible and intangible elements, the characteristics of which are quantified by the context and the logical descriptions in which they are incorporated. However, descriptive logic changes constantly, following the development of multiplicity and the extension of concepts. Therefore, the body of urban reality is redefined continuously following the change in both logical descriptions and contexts. Considering that each framework draws up an ideology and chooses to analyze its' core meaning, a linguistic analysis tool is developed for the combination of data visualization analysis, linguistic, and design methodologies to parameterize description of logics. Written speech is transformed into networks visualizing their ontological relationships. This creates a nebula of data (cluster) that assembles the immaterial reality of the urban fabric which is then transformed into local parameters by the appropriate methodological processes giving the relation between the nebula of descriptions and the locality of the network of descriptions. The material reality of the urban fabric is described through analyzing its' grid transformations. The infinite body ${ }^{1}$ of reality is synthesized through the composition of its' material and immaterial bodies.
\end{abstract}

Keywords: algorithmic design, conceptual design, construction of reality, linguistics, parametrization

\section{Reality: The Cosmic Dimension}

The objective of this research focuses on the construction of reality. The term reality is linked to pragmatism and derives from the ancient Greek word "pragma" and refers to anything that can be revealed through our senses ${ }^{2}$. In a continuous changing cosmos, the problematic of defining "pragma” or "object” has been addressed. The object has been characterized as "quasi" object intending to highlight the impossibility of the physical entities to be characterized by the continuous rearrangement of the logic of descriptions. Since the entity of "pragma" needs to be redefined and reality appears as a summary of those multiple entities, how can reality be addressed?

Eirini Krasaki, Dipl Architect Engineer, Ma, MSc., Ph.D. candidate, School of Architecture, Department of Architectural Language, Communication and Design, National Technical University of Athens, Athens, Greece.

1 The term body refers to a collection of information.

${ }^{2} \pi \rho \alpha ́ \gamma \mu \alpha$ (pragma) < ancient Greek $\pi \rho \tilde{a} \gamma \mu \alpha$, something that has been produced, created. 
This problematic of approaching and defining reality and furthermore the pragma or object has been addressed since the 4th BC by the allegory of Plato and the theory of ideas. In this allegory Plato described a group of individuals trapped in a cave, behind which there is a group of objects lighted by a fire. The sitting position of people allows them to only see the projections of the objects on the cave without being able to understand their complete geometry. Following their perception and senses they tend to believe that the truth is the continuously changing projection and not the actual entity. A more contemporary view of reality with clearer spatial implications is presented in the work of Andre Malraux The Imaginary Museum (1996). The author approaches the museum as a palimpsest, a dynamic archive of information, an open system where each imported information reorganizes and redefines the definition of the whole system. The subject within the complex of information is trying to capture the cluster of information that surrounds him in order to define the body of reality. Similar concepts have been used in the science fiction film $\operatorname{Tron}^{3}$. In this film the main character while trying to picture the cluster of information that moves within virtual space, enters to "another world" "another reality", the Grid, which defines his existing one. As architects, designers, contemporary thinkers within the digital era, how can we define the infinite body of reality? Can we use it as a design tool to define space, a mechanism or methodology which can give us unanticipated results?

\section{Reality: The Spatial Dimension}

To move from the cosmic dimension towards the spatial dimension, the concept of relativity is approached. As the relationship between two entities creates a spatial field, every form of material reality can be described by the concept of relativity. The spatial arrangement is defined by the development of forces between the entities of this field. Meanwhile the interaction of the two entities may create a third entity integrating the concept of emergence in the relational system. The entities consist of both material and immaterial elements and are connected through circuits or networks through which the phenomena are transmitted within the system (difference, change of the difference). Reality can be described as a system of associations composed of multiple interacting parts, which constitute a complex structure and are characterized by constant dependence and diversity. The parts of the system form collective behaviors that cannot be traced to individual parts of the system but are a result of their interactions and correlations. Therefore, a system that follows the characteristics of the complex systems is formed.

Complex systems are characterized by the connection, organization, and quantification of the variables that characterize their entities. Urban reality is described as such a system as it consists of both material and immaterial elements, actual and virtual, the characteristics of which are quantified by two main parameters: (a) by the context or framework and (b) thelogics of description to which they relate. As the logic of descriptions is changing constantly, following the development of multiplicity and the extension of concepts, the body of urban reality is continuously redefined.

In order to classify this system for this research the reality categorization proposed by mathematician Roger Penrose is applied. According to this, reality is classified to three categories: notional, physical, and mathematical—Platonical. Subsequently, methodologies to parameterize and to consecutively synthesize these categories are cited.

\footnotetext{
3 Tron is a 1982 American science fiction action-adventure film written and directed by Steven Lisberger from a story by Lisberger and Bonnie MacBird.
} 


\section{Rules of Notional Organization: The Framework}

The cluster of the notional-immaterial body of data, also referred as "nebula", is determined by an invisible structure, the framework. The framework determines the way the entities its' consists are perceived. What is not included within the framework cannot be understood; it is not referred to the current body of knowledge; it is classified as unknown.

The framework organizes entities in a specified way, incorporating them into a structured system of relations. It is part of what is called the "cognitive unconscious", which is inaccessible to the conscious mind, but it determines our decisions, our actions, and the way we process data. Referring again to the allegory of Plato this framework can be correlated to the field of vision that guides the accessibility to knowledge and information of the individuals. As the invisible structure of knowledge changes, the framework and the logics of description follow this change. In this research myth (either in its primary meaning or by its' evolution to what we call today social media) is considered as the dominant factor for the formation of the "cognitive unconsciousness" and furthermore the logics of description. Myth is linked to the way a culture identifies and gives meaning to the world that surrounds it. By analyzing both modern fictions and traditional myths, a tool to classify the notional organization of reality is formed.

\section{Rules of Physical Organization: The Form}

Form is perceived as a diagram of forces, as the end result of the interaction between the particles of the diagram and the momentary equilibrium between those forces. The constant change of the logics of description has led to the de-stabilization of the object. The form is considered as a nebula of potential states that vary in relation to time and logics of descriptions. Virtual is a mess of constraints, a dynamic state that is resolved with the actual in a different way every time.

In order to analyze form, grid is used as a tool. The grid forms the primary systemic organization of form. A diagram remains dominant throughout history, the expression of which changes over time following the change of the logics of description. The Cartesian grid of the past, a characteristic of modernism, an emblem of industrialization, reflects the concepts of standardization and mass production. Its characteristics are homogeneity, the constant form (repetition) based on the Euclidean geometry and the available tools of the given time. In a more contemporary organization, we can see the grid as a field of potentialities, shaped in relation to the forces responding to local conditions, a malleable network that can be easily used to produce non-standard elements and massively personalized spaces. The ordinary Cartesian grid can be neutral and rigid but by parametrizing its' nodes according to data and information it can be programmed to deform in multiple ways. The grid will form the basic tool for the parameterization of form. Therefore, the body of reality consists of the pre-referred three main parts (notional, physical, and the connections between them). For the parameterization of the notional the analysis of the logical description will be used and for the parameterization of the material the analysis of the grid constitutes the system of form. The synthesis of these three systems will construct the body of reality.

\section{The Paradigm: Eleusis}

\section{The Methodology for Analyzing the Body of Immaterial Data}

Eleusis is a palimpsest of descriptions that have been synthesized chronologically and spatially in multiple frameworks. Under this light, it is considered as an appropriate example for the pilot implementation of the 
proposed design methodology. For the implementation of the methodology, it is necessary to detect the dominant frameworks that have been shaped over time. For this reason, the main historical events are disclosed. The event is defined as the situation which does not follow the rules of the system, an "intervention" that overrides the pre-existing situation. In this particular paradigm six historical "events" are revealed. In this research it is assumed that the system of reality is in an equilibrium state before and after the occurrence of the event. For the analysis of the notional complexes during these six historical periods and in order to parameterize both the logics of descriptions and myths, the tool for analyzing written language is used.

The above-mentioned mechanism converts the written text into a network, matching every word to a node of the network (after having deducted those words that do not add value to the meaning of the text). Then the network nodes are programmed to join each other in such a way that the factor of time is deducted from the networking of the written language in order to map the ontological relations of the network and not the relation of time. The resulting network is a dialectical image that has been removed from the historical continuum and has been integrated into the time of the particular snapshot. This creates a nebula of the notional reality for each of the six snapshots. Although this cluster or nebula cannot be linked to spatial coordinates, it appears the topological relations between the nodes. The nebula is scanned in order to reveal those nodes that can be linked to landmarks or other spatial coordinates. The nebula is reorganized and converted to a local description using an algorithm that works accordingly to the attraction of springs within the network. Each network connection is a string between its two nodes. The force value is given by the weight of the network, the repetition number of the word within the text. The stabilization of the springs gives us the balance of the nebula of the descriptions in relation to the locality and the networking of the written word. In order to connect this nebula of data to spatial characteristics, the words that are referred to a landmark are defined by its' spatial dimensions and stabilize this node of the network to its' exact coordinates. Each point corresponds to a logic of description, in a context in which it is also related to in some hyper local links. Considering that each framework draws up an ideology, and it has been chosen to analyze its' central meaning, we search the dominant concepts or metaphors that synthesize it. A parameterization of the framework is formed. For example, the field of the archaeological site contains within its limit's descriptions of Homer, Pausanias which are described within the sanctuary framework in the light of paganism. At the same time, it is hyper-locally linked to the five holy cities. This field refers to a set of attributes corresponding to the variety of the related descriptions of logic such as Plato, plateau, Themistocles, Athena, immortality, ceremony, Neptune, etc. Concluding, for this field we have the set of attributes resulting from the descriptions of logic within which it is identified. The complex of reality of this field will be synthesized using these logical descriptions.

\section{The Methodology for Analyzing the Body of Material Data}

In order to determine the morphological shape, the urban fabric is used as a mechanism of analysis, since it is perceived as the mechanism for classifying the entities and properties of the object within space. For analyzing the urban grid, the tools from the theory of Space Syntax ${ }^{4}$ are used, a theory of spatial analysis whose characteristics is the emphasis from geometry and morphology to syntax, i.e., on the spatial arrangements and relationships. In this research it is considered as an appropriate toolset as it approaches the morphology under the relativity system through which the construction of reality is examined.

\footnotetext{
${ }^{4}$ A set of theories and techniques for the analysis of spatial configurations conceived by Bill Hillier and Julienne Hanson in the late 1970 s to early 1980 s.
} 
In the urban fabric of Eleusis, the basic different morphological assemblages ${ }^{5}$ are established and later analyzed by this tool. The urban grid is analyzed in terms of its elasticity.

Subsequently, the notional data from the previous analysis are corresponded to the spatial coordinates. It is observed that the urban webs associated with numerous logical descriptions bear the most distinct changes in the morphology of their urban fabric.

The above is an outline to the approach, analysis, and methodology to analyze the complexity of the urban body in the light of the multiplicity of logical descriptions and their integration into multiple contexts. Considering that the body of reality is constituted by infinite descriptions and the infinity of these descriptions is represented by multidimensional vectors, then the representations of the body we see are only a projection of this multiplicity in the urban fabric. The definition of the infinity of the body is subject to further work and research. The multiplicity of digital tools that we have access to and the interdisciplinary of media are offering new grounds for thought and not just composition. Perhaps we are in the transition to a new paradigm that which Carpo calls a "second shift in the digital world" and the interpretation we give to the body of reality occurs within this framework.

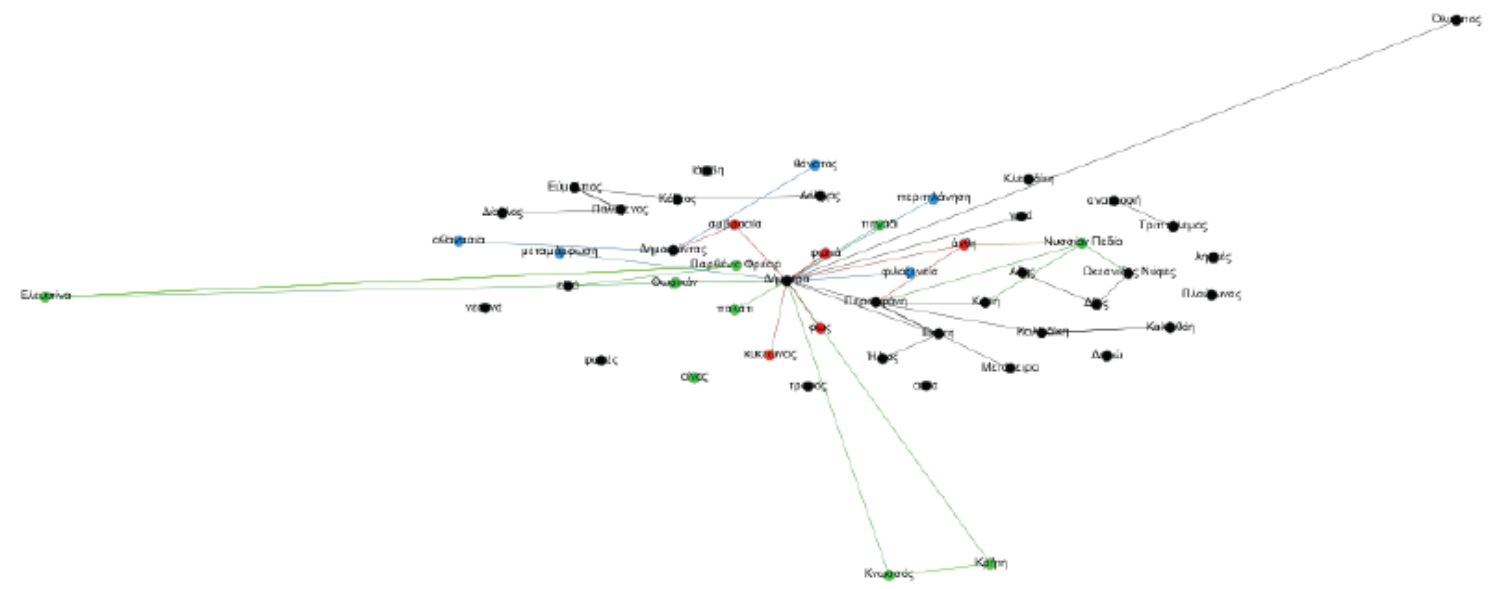

figure 01

Figure 1. Nebula of attributes corresponding to the logic of descriptions related to the specific place.

\footnotetext{
${ }^{5}$ French: Agencement, is an ontological framework developed by Gilles Deleuze and Félix Guattari, presented in their book $A$ Thousand Plateaus (1980).
} 


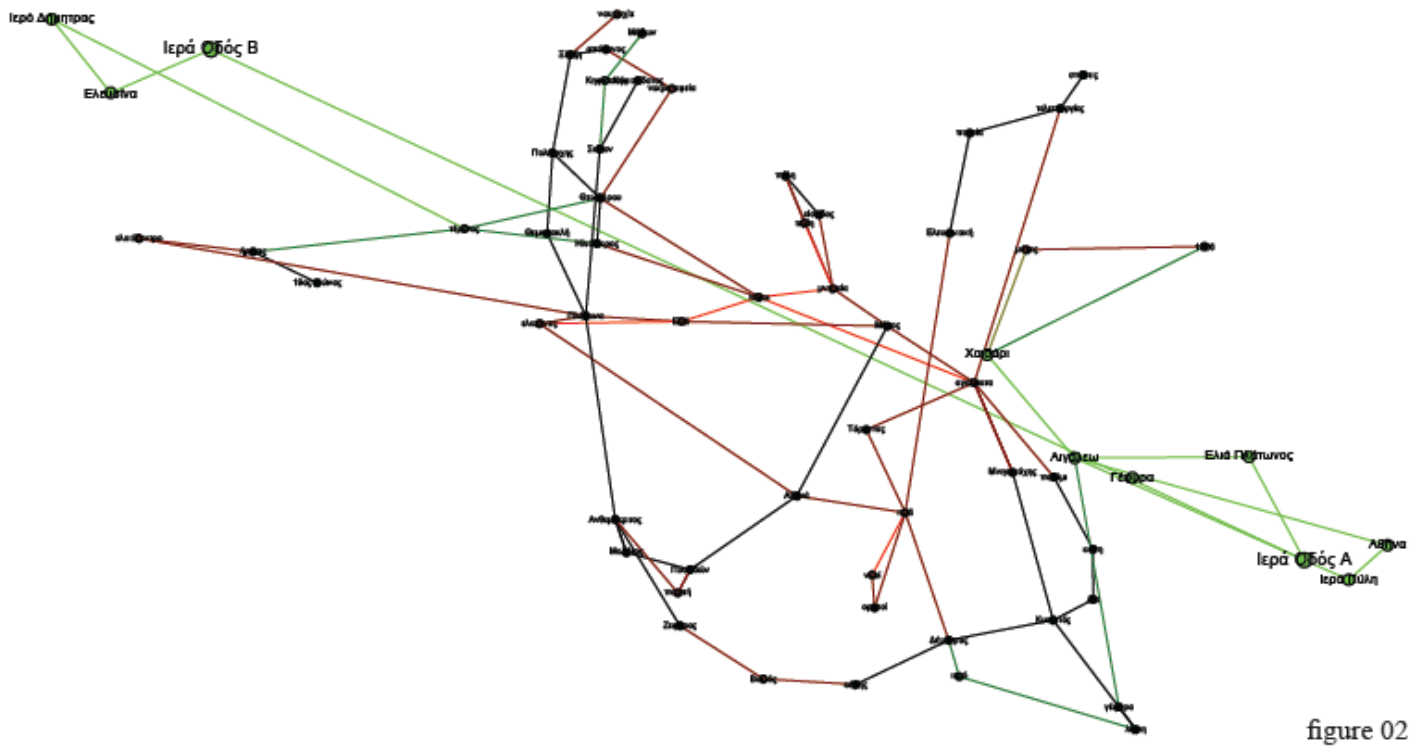

Figure 2. Nebula of attributes corresponding to the logic of descriptions related to the specific place and its hyper topical network.

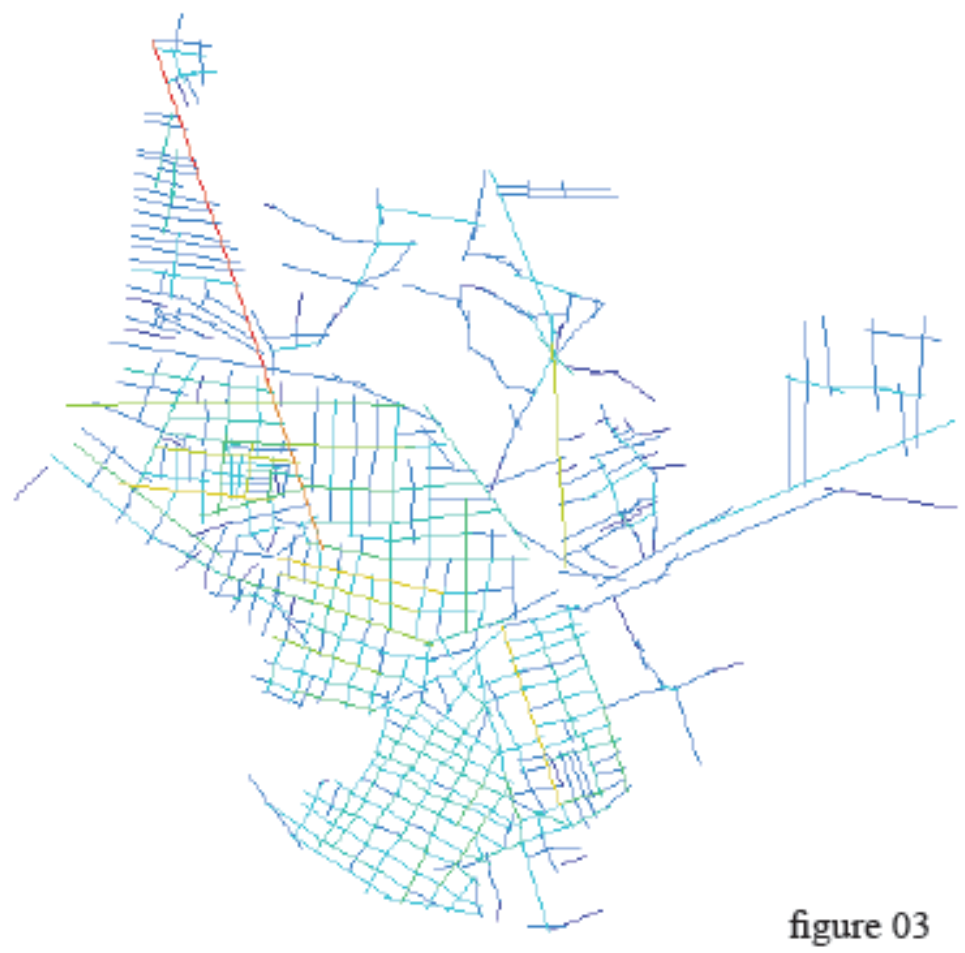

Figure 3. Appraisal of grid elasticity using Space Syntax tools. 


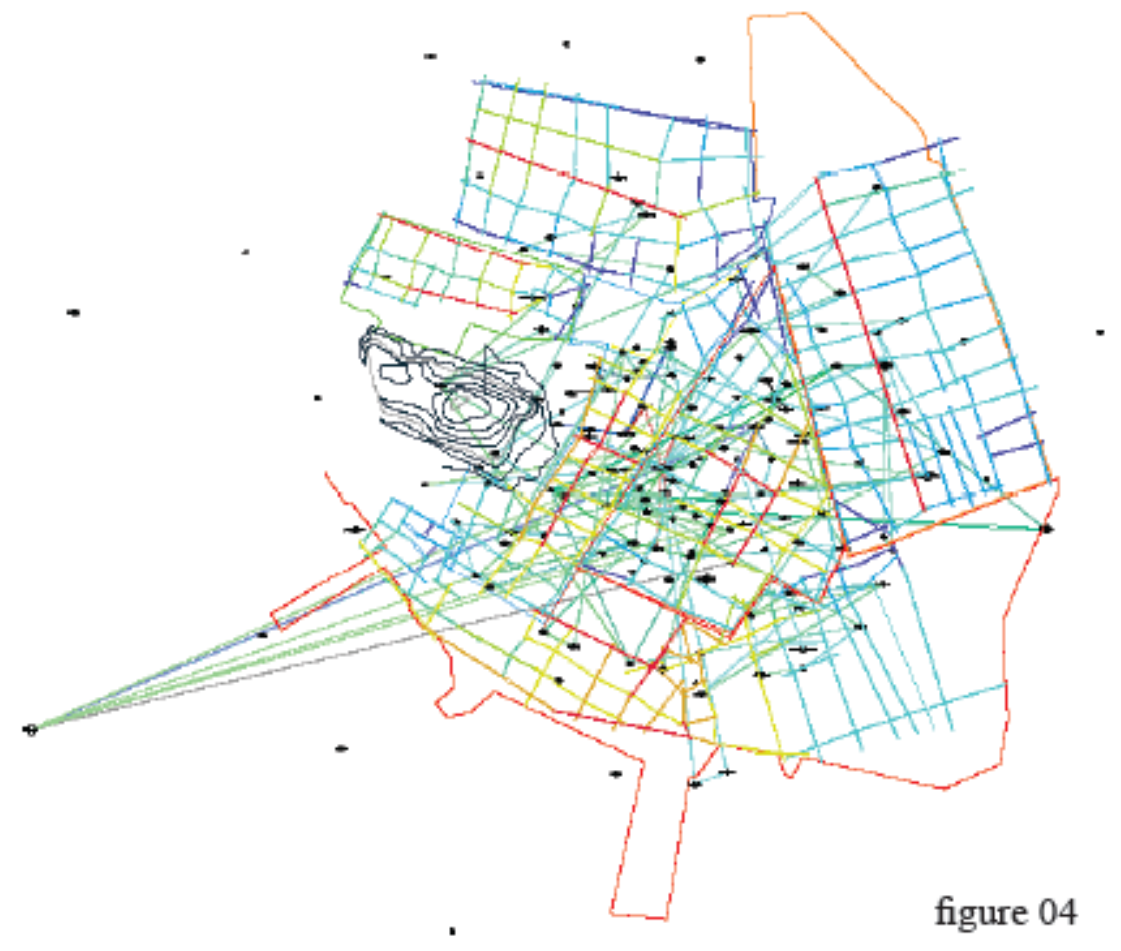

Figure 4. Correlation between spatial coordination (points), the attributes from the corresponding logic of descriptions, and the elasticity of the urban grid.

\section{References}

Carpo, M. (2017). The second digital turn, design beyond intelligence. Cambridge Massachusetts: MIT Press.

Malraux, A. (1996). La Musee Imaginaire. Paris: Gallimard Education.

Papalexopoulos, D. (2008). Digital regionalism. Athens: Libro.

Parmenides, G. (2017). The metaphor and the application of topological attributes: Framing and reframing the design object. Retrieved from http://www.arch.ntua.gr/node-resources1153\#resource-11237

Penrose, R. (2004). The road to reality, a complete guide to the laws of universe. Athens: Politeia. 